\title{
Optimization of flue gas turbulent heat transfer with condensation in a tube
}

\author{
SONG WeiMing, MENG JiAn \& LI ZhiXin* \\ Key Laboratory for Thermal Science and Power Engineering of Ministry of Education, Department of Engineering Mechanics, Tsinghua Univer- \\ sity, Beijing 100084, China
}

Received January 24, 2011; accepted March 14, 2011

\begin{abstract}
Conservation equations for sensible and latent entransy are established for flue gas turbulent heat transfer with condensation in a tube, and the entransy dissipation expression is deduced. The field synergy equation is obtained on the basis of the extremum entransy dissipation principle for flue gas turbulent heat transfer with condensation. The optimal velocity field is numerically obtained by solving the field synergy equation. The results show that the optimal velocity field contains multiple longitudinal vortices near the tube surface. These improve the synergy not only between the velocity and temperature fields but also between the velocity and vapor concentration fields. Therefore, the turbulent heat and mass transfers are significantly enhanced.
\end{abstract}

condensation, entransy, entransy dissipation, field synergy, longitudinal vortex

Citation: $\quad$ Song W M, Meng J A, Li Z X. Optimization of flue gas turbulent heat transfer with condensation in a tube. Chinese Sci Bull, 2011, 56: 1978-1984, doi: 10.1007/s11434-011-4533-9

In condensation-type boilers using natural gas, the mass fraction of vapor in the flue gas is about $7 \%$ to $25 \%$. Tubular heat exchangers are often used to recover the latent heat of the vapor, resulting in a higher thermal efficiency [1]. In the tubular heat exchangers, the vapor condenses on the tube surface if the local tube surface temperature is lower than the dew point. Therefore, there is both sensible heat transfer via the temperature difference and latent heat transfer via the vapor concentration difference during condensation for turbulent heat transfer with vapor condensation. There has been much research [2-7] on turbulent heat transfer with condensation in which the effects of Reynolds number, tube surface temperature and mass fraction of the vapor have been investigated. However, there has been little theoretical study for the purpose of understanding the heat transfer mechanism.

Bejan [8] analyzed convective heat transfer, considering entropy generation based on irreversible thermodynamics, and optimized the convective heat transfer according to the

*Corresponding author (email: lizhx@tsinghua.edu.cn) minimum entropy generation (MEG) principle. However, the heat transfer rate should be of much greater interest during heat transfer. Guo et al. $[9,10]$ introduced a physical quantity describing the heat transfer ability of an object, entransy, which is defined as half the product of internal energy and temperature of the object. The extremum entransy dissipation (EED) principle was also established for heat transfer optimization. There has been much work over recent years on heat transfer optimization based on entransy dissipation [11-16] and the optimization results [17-22] have shown that the EED principle is superior to the MEG principle for heat transfer optimization.

On the basis of the EED principle, Meng et al. [23] deduced the field synergy equation for laminar convective heat transfer, using the variation principle for given input viscous dissipation. Their numerical results showed that the optimal velocity field was a multiple longitudinal vortex flow, and a new enhanced tube was developed to generate multiple longitudinal vortices. Song et al. [24] obtained the optimal velocity field for laminar convective heat transfer in a rectangular channel. The heat transfer was significantly 
enhanced by longitudinal vortex flow. Chen et al. [25] deduced the field synergy equation for turbulent heat transfer and obtained the optimal velocity field with small eddies near the boundary for a 2D parallel plate channel. Chen et al. [26] introduced the conservation equations for sensible entransy and latent entransy for the evaporative cooling system. Moisture entransy and moisture entransy dissipation were defined. Song et al. [27] defined entransy and entransy dissipation and deduced the field synergy equation for laminar convective heat transfer with vapor condensation. An optimal velocity field with multiple longitudinal vortices was obtained numerically.

In this study, the flue gas in the tube is considered as a mixture of vapor and non-condensable gas. The flue gas flow in the tube is turbulent, with Reynolds numbers between 5000 and 16000. First, entransy and entransy dissipation are defined for the turbulent heat transfer with vapor condensation. Second, the field synergy equation for turbulent convective heat transfer with condensation is deduced using the EED principle. Third, the optimal velocity field is numerically obtained for given time-averaged viscous dissipation.

\section{Entransy and entransy dissipation}

Similar to laminar convective heat transfer with vapor condensation [27], entransy conservation equations will be established and the entransy dissipation expression deduced for turbulent heat transfer with condensation. Figure 1 shows the flue gas turbulent heat transfer with condensation in a vertical tube. The vapor at high temperature condenses on the tube surface at low temperature.

The flue gas turbulent heat transfer must satisfy the governing equations.

Time-averaged energy equation:

$$
\rho U \cdot \nabla\left(c_{p} T\right)=\nabla \cdot\left(\lambda_{\mathrm{eff}} \nabla T\right)
$$

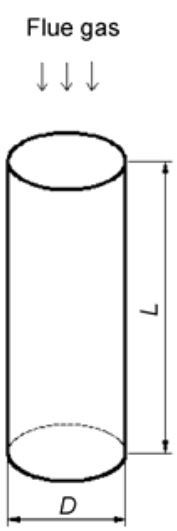

Figure 1 Flue gas turbulent heat transfer with condensation in a vertical tube. where $\rho, c_{p}$ and $\lambda_{\text {eff }}$ are the density, specific heat and effective thermal conductivity of the flue gas, respectively. $U$ is the velocity vector and $T$ is the temperature. Eq. (1) is also the sensible heat conservation equation for the flue gas.

The time-averaged species equation is

$$
\rho U \cdot \nabla \omega=\nabla \cdot\left(\rho D_{\text {eff }} \nabla \omega\right),
$$

where $\omega$ is the mass fraction of the vapor and $D_{\text {eff }}$ is the effective mass diffusion coefficient of the vapor in the flue gas.

The vaporization latent heat of water, $\gamma$, is assumed to be constant. Multiplying both sides of eq. (2) by $\gamma$ gives the latent heat conservation equation:

$$
\rho U \cdot \nabla(\gamma \omega)=\nabla \cdot\left[\rho D_{\text {eff }} \nabla(\gamma \omega)\right] .
$$

Multiplying both sides of eq. (1) by $T$ gives the conservation equation for the sensible heat entransy:

$$
\rho U \cdot \nabla g_{\mathrm{s}}=\nabla \cdot\left(\lambda_{\mathrm{eff}} T \nabla T\right)-\lambda_{\mathrm{eff}}(\nabla T)^{2},
$$

where $g_{s}=c_{p} T^{2} / 2$ is the sensible heat entransy per unit mass of the flue gas. The sensible heat entransy describes the sensible heat transfer ability of the flue gas. $g_{\mathrm{s}, \mathrm{dis}}=$ $\lambda_{\text {eff }}(\nabla T)^{2}$ is the sensible heat entransy dissipation. Eq. (4) indicates that the sensible heat entransy rate via convection equals that via conduction minus the sensible heat entransy dissipation rate.

Likewise, multiplying both sides of eq. (3) by $T$ gives

$$
\begin{aligned}
T \rho U \cdot \nabla(\gamma \omega)= & \nabla \cdot\left[\rho D_{\mathrm{eff}} T \nabla(\gamma \omega)\right] \\
& -\rho D_{\mathrm{eff}} \nabla T \cdot \nabla(\gamma \omega) .
\end{aligned}
$$

Assuming a linear relationship between the mass fraction of the vapor and its corresponding dew point, $T_{\mathrm{dp}}=a \omega+b$, eq. (5) can be written as the conservation equation for the latent heat entransy:

$$
\begin{aligned}
\rho U \cdot \nabla g_{1}= & \nabla \cdot\left[\rho D_{\text {eff }} T \nabla(\gamma \omega)\right] \\
& -\rho D_{\text {eff }} \nabla T \cdot \nabla(\gamma \omega) \\
& -\left(T-T_{\mathrm{dp}}\right) \nabla \cdot\left[\rho D_{\text {eff }} \nabla(\gamma \omega)\right],
\end{aligned}
$$

where $g_{1}=\gamma \omega T_{\mathrm{dp}} / 2$ is the latent heat entransy per unit mass of the flue gas. The latent heat entransy describes the latent heat transfer ability of the flue gas. The left side of eq. (6) is the latent heat entransy in the flue gas flow, while the first item on the right side of eq. (6) represents the latent heat entransy diffusion induced by vapor diffusion. The second item is the latent heat entransy dissipation associated with not only the vapor concentration field but also the temperature field. The last item is the latent heat entransy diffusion due to the difference between the flue gas temperature and dew point. 
Combining eqs. (4) and (6) yields the entransy conservation equation for the flue gas turbulent heat transfer:

$$
\begin{aligned}
\rho U \cdot \nabla g= & \nabla \cdot\left[\lambda_{\mathrm{eff}} T \nabla T+\rho D_{\mathrm{eff}} T \nabla(\gamma \omega)\right]-g_{\mathrm{dis}}, \\
g_{\mathrm{dis}}= & \lambda_{\mathrm{eff}}(\nabla T)^{2}+\rho D_{\mathrm{eff}} \nabla T \cdot \nabla(\gamma \omega) \\
& +\left(T-T_{\mathrm{dp}}\right) \nabla \cdot\left[\rho D_{\mathrm{eff}} \nabla(\gamma \omega)\right],
\end{aligned}
$$

where $g=\left(c_{p} T^{2}+\gamma \omega T_{\mathrm{dp}}\right) / 2$, a measure of the total heat transfer ability of the flue gas, is the entransy per unit mass, and $g_{\text {dis }}$ is the total entransy dissipation.

\section{Field synergy equation}

Similar to optimizing single-phase convective heat transfer [23-25], and laminar convective heat transfer with condensation [27], the optimization objective for the turbulent heat transfer with condensation is to determine the optimal velocity field that leads to the EED for given time-averaged viscous dissipation. Meanwhile, the flow and heat transfer are also governed by the continuity, time-averaged energy and species equations. This is a typical functional extremum problem. Employing the calculus of variations, a Lagrange function is constructed with the given constraints:

$$
\Pi=\iiint_{\Omega}\left\{\begin{array}{l}
g_{\text {dis }}+C_{0} \phi_{\mathrm{t}}+A\left[\nabla \cdot\left(\lambda_{\text {eff }} \nabla T\right)-\rho c_{p} U \cdot \nabla T\right] \\
+B\left[\nabla \cdot\left(\rho D_{\text {eff }} \nabla \omega\right)-\rho U \cdot \nabla \omega\right]+C \nabla \cdot(\rho U)
\end{array}\right\} \mathrm{d} V,(9)
$$

where $C_{0}, A, B$ and $C$ are Lagrange multipliers. Owing to the different types of constraints, $C_{0}$ remains constant for given time-averaged viscous dissipation, while $A, B$ and $C$ are functions of $U, T, \omega$ and local position. $\phi_{\mathrm{t}}=\phi\left(\mu+\mu_{\mathrm{t}}\right) / \mu$, is the time-averaged viscous dissipation rate per unit volume. The zero-equation model $[25,28,29]$ is used to calculate the turbulent viscosity.

$$
\mu_{\mathrm{t}}=0.03874 \rho|U| l,
$$

where $l$ is the distance to the nearest wall. The zero-equation model can be considered a reasonable model for predicting turbulent flow with reasonable results.

The variation in $\Pi$ with respect to $T$ gives the governing equation of $A$ :

$$
-\rho c_{p} U \cdot \nabla A=\nabla \cdot\left(\lambda_{\text {eff }} \nabla A\right)-2 \nabla \cdot\left(\lambda_{\text {eff }} \nabla T\right) .
$$

The boundary conditions are $A=0$ for given boundary temperatures and $\partial A / \partial n=2 \partial T / \partial n+\rho D_{\text {eff }} \gamma / \lambda_{\text {eff }} \partial \omega / \partial n$ for given boundary heat flux.

The variation in $\Pi$ with respect to $\omega$ gives the governing equation for $B$ :

$$
-\rho U \cdot \nabla B=\nabla \cdot\left(\rho D_{\text {eff }} \nabla B\right)-2 a \nabla \cdot\left(\rho D_{\text {eff }} \gamma \nabla \omega\right) .
$$

The boundary conditions are $B=-\gamma\left(T-T_{\mathrm{dp}}\right)$ for given bound- ary species and $\partial B / \partial n=a \gamma \partial \omega / \partial n$ for a given boundary species gradient.

The variation in $\Pi$ with respect to velocity component $u$ is

$$
\begin{aligned}
& \nabla \cdot\left(\mu_{\mathrm{eff}} \nabla u\right)+\frac{\rho c_{p} A}{2 C_{0}} \frac{\partial T}{\partial x}+\frac{\rho B}{2 C_{0}} \frac{\partial \omega}{\partial x} \\
& +\frac{\rho}{2 C_{0}} \frac{\partial C}{\partial x}-\frac{\sigma u}{2 C_{0} P r_{\mathrm{t}}}\left[(\nabla T)^{2}+A \nabla^{2} T\right] \\
& -\frac{\gamma \sigma u}{2 C_{0} S c_{\mathrm{t}}} \nabla T \nabla \omega-\frac{\left[\left(T-T_{\mathrm{dp}}\right) \gamma+B\right] \sigma u}{2 C_{0} S c_{\mathrm{t}}} \nabla^{2} \omega \\
& -\frac{\sigma u \phi_{\mathrm{t}}}{2 \mu_{\mathrm{eff}}}+\left(\frac{\partial \mu_{\mathrm{eff}}}{\partial x} \frac{\partial u}{\partial x}+\frac{\partial \mu_{\mathrm{eff}}}{\partial y} \frac{\partial v}{\partial x}+\frac{\partial \mu_{\mathrm{eff}}}{\partial z} \frac{\partial w}{\partial x}\right) \\
& -\frac{\sigma}{2 C_{0}}\left[\frac{A}{P r_{\mathrm{t}}} \frac{\partial T}{\partial x}+\frac{\left(T-T_{\mathrm{dp}}\right) \gamma+B}{S c_{\mathrm{t}}} \frac{\partial \omega}{\partial x}\right] \\
& \times\left[\frac{u}{l} \frac{\partial l}{\partial x}-\left(u \frac{\partial u}{\partial x}+v \frac{\partial v}{\partial x}+w \frac{\partial w}{\partial x}\right) \frac{u}{|U|^{2}}+\frac{\partial u}{\partial x}\right] \\
& -\frac{\sigma}{2 C_{0}}\left[\frac{A}{P r_{\mathrm{t}}} \frac{\partial T}{\partial y}+\frac{\left(T-T_{\mathrm{dp}}\right) \gamma+B}{S c_{\mathrm{t}}} \frac{\partial \omega}{\partial y}\right] \\
& \times\left[\frac{u}{l} \frac{\partial l}{\partial y}-\left(u \frac{\partial u}{\partial y}+v \frac{\partial v}{\partial y}+w \frac{\partial w}{\partial y}\right) \frac{u}{|U|^{2}}+\frac{\partial u}{\partial y}\right] \\
& -\frac{\sigma}{2 C_{0}}\left[\frac{A}{P r_{\mathrm{t}}} \frac{\partial T}{\partial z}+\frac{\left(T-T_{\mathrm{dp}}\right) \gamma+B}{S c_{\mathrm{t}}} \frac{\partial \omega}{\partial z}\right] \\
& \times\left[\frac{u}{l} \frac{\partial l}{\partial z}-\left(u \frac{\partial u}{\partial z}+v \frac{\partial v}{\partial z}+w \frac{\partial w}{\partial z}\right) \frac{u}{|U|^{2}}+\frac{\partial u}{\partial z}\right] \\
& +\frac{1}{2 C_{0}} \frac{\partial}{\partial x}\left\{\left[\frac{A}{P r_{\mathrm{t}}} \frac{\partial T}{\partial x}+\frac{\left(T-T_{\mathrm{dp}}\right) \gamma+B}{S c_{\mathrm{t}}} \frac{\partial \omega}{\partial x}\right] \sigma u\right\} \\
& +\frac{1}{2 C_{0}} \frac{\partial}{\partial y}\left\{\left[\frac{A}{P r_{\mathrm{t}}} \frac{\partial T}{\partial y}+\frac{\left(T-T_{\mathrm{dp}}\right) \gamma+B}{S c_{\mathrm{t}}} \frac{\partial \omega}{\partial y}\right] \sigma u\right\} \\
& +\frac{1}{2 C_{0}} \frac{\partial}{\partial z}\left\{\left[\frac{A}{P r_{\mathrm{t}}} \frac{\partial T}{\partial z}+\frac{\left(T-T_{\mathrm{dp}}\right) \gamma+B}{S c_{\mathrm{t}}} \frac{\partial \omega}{\partial z}\right] \sigma u\right\}=0,
\end{aligned}
$$

where $\sigma=0.03874 p l|U|^{-1}$. The variations in $\Pi$ with respect to velocity components $v$ and $w$ are similar to eq. (13).

Comparing eq. (13) with the momentum equation in the $x$ coordinate

$$
\rho U \cdot \nabla u=-\partial P / \partial x+\nabla \cdot\left(\mu_{\mathrm{eff}} \nabla u\right)
$$

gives

$$
C=-2 C_{0} P / \rho,
$$

where $C_{0}$ is related to the time-averaged viscous dissipation. 
Eq. (13) can be rewritten as

$$
\rho U \cdot \nabla u=-\partial P / \partial x+\nabla \cdot\left(\mu_{\mathrm{eff}} \nabla u\right)+F_{x},
$$

in which

$$
\begin{aligned}
& F_{x}=\rho U \cdot \nabla u+\frac{\rho c_{p} A}{2 C_{0}} \frac{\partial T}{\partial x} \\
& +\frac{\rho B}{2 C_{0}} \frac{\partial \omega}{\partial x}-\frac{\sigma u}{2 C_{0} P r_{\mathrm{t}}}\left[(\nabla T)^{2}+A \nabla^{2} T\right] \\
& -\frac{\gamma \sigma u}{2 C_{0} S c_{\mathrm{t}}} \nabla T \nabla \omega-\frac{\left[\left(T-T_{\mathrm{dp}}\right) \gamma+B\right] \sigma u}{2 C_{0} S c_{\mathrm{t}}} \nabla^{2} \omega \\
& -\frac{\sigma u \phi_{\mathrm{t}}}{2 \mu_{\mathrm{eff}}}+\left(\frac{\partial \mu_{\mathrm{eff}}}{\partial x} \frac{\partial u}{\partial x}+\frac{\partial \mu_{\mathrm{eff}}}{\partial y} \frac{\partial v}{\partial x}+\frac{\partial \mu_{\mathrm{eff}}}{\partial z} \frac{\partial w}{\partial x}\right) \\
& -\frac{\sigma}{2 C_{0}}\left[\frac{A}{P r_{\mathrm{t}}} \frac{\partial T}{\partial x}+\frac{\left(T-T_{\mathrm{dp}}\right) \gamma+B}{S c_{\mathrm{t}}} \frac{\partial \omega}{\partial x}\right] \\
& \times\left[\frac{u}{l} \frac{\partial l}{\partial x}-\left(u \frac{\partial u}{\partial x}+v \frac{\partial v}{\partial x}+w \frac{\partial w}{\partial x}\right) \frac{u}{|U|^{2}}+\frac{\partial u}{\partial x}\right] \\
& -\frac{\sigma}{2 C_{0}}\left[\frac{A}{P r_{\mathrm{t}}} \frac{\partial T}{\partial y}+\frac{\left(T-T_{\mathrm{dp}}\right) \gamma+B}{S c_{\mathrm{t}}} \frac{\partial \omega}{\partial y}\right] \\
& \times\left[\frac{u}{l} \frac{\partial l}{\partial y}-\left(u \frac{\partial u}{\partial y}+v \frac{\partial v}{\partial y}+w \frac{\partial w}{\partial y}\right) \frac{u}{|U|^{2}}+\frac{\partial u}{\partial y}\right] \\
& -\frac{\sigma}{2 C_{0}}\left[\frac{A}{P r_{\mathrm{t}}} \frac{\partial T}{\partial z}+\frac{\left(T-T_{\mathrm{dp}}\right) \gamma+B}{S c_{\mathrm{t}}} \frac{\partial \omega}{\partial z}\right] \\
& \times\left[\frac{u}{l} \frac{\partial l}{\partial z}-\left(u \frac{\partial u}{\partial z}+v \frac{\partial v}{\partial z}+w \frac{\partial w}{\partial z}\right) \frac{u}{|U|^{2}}+\frac{\partial u}{\partial z}\right] \\
& +\frac{1}{2 C_{0}} \frac{\partial}{\partial x}\left\{\left[\frac{A}{P r_{\mathrm{t}}} \frac{\partial T}{\partial x}+\frac{\left(T-T_{\mathrm{dp}}\right) \gamma+B}{S c_{\mathrm{t}}} \frac{\partial \omega}{\partial x}\right] \sigma u\right\} \\
& +\frac{1}{2 C_{0}} \frac{\partial}{\partial y}\left\{\left[\frac{A}{P r_{\mathrm{t}}} \frac{\partial T}{\partial y}+\frac{\left(T-T_{\mathrm{dp}}\right) \gamma+B}{S c_{\mathrm{t}}} \frac{\partial \omega}{\partial y}\right] \sigma u\right\} \\
& +\frac{1}{2 C_{0}} \frac{\partial}{\partial z}\left\{\left[\frac{A}{P r_{\mathrm{t}}} \frac{\partial T}{\partial z}+\frac{\left(T-T_{\mathrm{dp}}\right) \gamma+B}{S c_{\mathrm{t}}} \frac{\partial \omega}{\partial z}\right] \sigma u\right\} \text {. }
\end{aligned}
$$

Eq. (16) is the $x$-coordinate in Euler's equation governing the fluid velocity and temperature fields under the EED principle during turbulent heat transfer with condensation. Essentially, it is the $x$-coordinate time-averaged momentum equation with a special additional volume force $F_{x}$, also called the $x$-coordinate field synergy equation. The field synergy equations in the $y$ - and $z$-coordinates are similar to eq. (16). These additional volume forces improve the synergy not only between the velocity and temperature fields but also between the velocity and vapor concentration fields. Therefore, the turbulent heat transfer is enhanced for given time-averaged viscous dissipation.

For given boundary conditions, the continuity, field synergy, energy and species equations and eqs. (11) and (12), including the six variables $U, T, \omega, P, A$ and $B$, can be solved together to provide the optimal velocity field, which gives the EED for given time-averaged viscous dissipation.

\section{Numerical model}

Figure 1 shows the flue gas turbulent heat transfer in a vertical tube with diameter $D=40 \mathrm{~mm}$ and length $L=60 \mathrm{~mm}$. The turbulent heat transfer with condensation can be numerically optimized using the field synergy equation given above. There are four assumptions in the numerical model.

(i) Neglecting the presence of droplets and liquid film implies, in principle, that the condensate is promptly removed from the tube surface.

(ii) When the local tube surface temperature is lower than the corresponding dew point, there is condensation and the saturation value of the mass fraction is utilized as a Dirichlet boundary condition for the species conservation equation. In addition, the ideal gas relationship is used to compute the mass fraction of the vapor corresponding to the saturation pressure at the absolute tube surface temperature.

$$
\omega_{\mathrm{w}}=P_{\mathrm{s}} /\left(\rho R_{\mathrm{g}, \mathrm{v}} T_{\mathrm{w}}\right),
$$

where $R_{\mathrm{g}, \mathrm{v}}$ is the gas constant of the vapor, and the approximate relationship used to evaluate the saturation pressure is

$$
P_{\mathrm{s}}=610.78 \exp \left[17.2694\left(T_{\mathrm{w}}-273.15\right) /\left(T_{\mathrm{w}}-34.58\right)\right] .
$$

(iii) The transverse velocity component induced by the condensation is disregarded. Therefore, the no-slip condition is used for the tube surface.

(iv) When there is condensation, the amounts of both flue gas and vapor are reduced and latent heat is released. Therefore, in the numerical model, the mass, species and energy sources are added to the boundary cells where the vapor condenses. The boundary cells are shown in Figure 2. The mass and species sources are $S_{\mathrm{m}}=-m_{\mathrm{v}} A_{\text {face }} / V_{\text {cell }}$ and the energy source is $S_{\mathrm{e}}=m_{\mathrm{v}} \gamma A_{\text {face }} / V_{\text {cell }} \cdot m_{\mathrm{v}}=-\rho D(\partial \omega / \partial n)_{\mathrm{w}}$ is the condensing rate of the vapor. $A_{\text {face }}$ is the interface area of the boundary cell and $V_{\text {cell }}$ is the boundary cell volume.

In the simulation, the flue gas temperature, velocity and mass fraction of the vapor are given at the inlet. $T_{\mathrm{in}}=340 \mathrm{~K}$, $R e=10000, \omega=10 \%$. The tube surface temperature is constant; $T_{\mathrm{w}}=300 \mathrm{~K}$. Fully developed distributions of variables $A$ and $B$ are assumed at the outlet, extended $300 \mathrm{~mm}$ downstream with zero gauge pressure.

The commercial computational fluid dynamics code FLUENT6.0 was used to solve the governing equations. The SIMPLEC algorithm was used for the velocity-pressure 


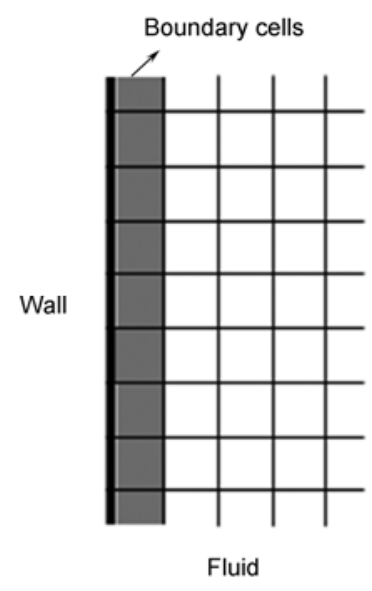

Figure 2 Boundary cells in the simulation.

coupling. The QUICK scheme was employed for the discretization of the convection and diffusion terms. A userdefined function was used for varying properties of the flue gas with different temperature, adding the additional volume force in the momentum equation, governing equations and corresponding boundary conditions of variables $A$ and $B$, species boundary conditions of the vapor on the tube surface and adding sources to the boundary cells.

\section{Optimization results}

The turbulent heat transfer with condensation in the vertical tube was numerically optimized. The total time-averaged viscous dissipation, total heat transfer rate and condensing heat transfer rate before and after optimization with $C_{0}=-1.0 \times 10^{6} \mathrm{~K}$ are listed in Table 1 .

Table 1 shows that, compared with the case before optimization, the total time-averaged viscous dissipation of the flue gas flow in the vertical tube after optimization increased by $116.2 \%$, while the total heat transfer rate increased by $30.6 \%$ and the condensing heat transfer rate increased by $41.9 \%$. The heat transfer rate and viscous dissipation increments in this case are representative of practical turbulent heat transfer enhancement. The simulation results were similar for different values of $C_{0}$, which is related to the viscous dissipation. Therefore, both the sensible and latent heat transfers were enhanced after optimization. In the optimization of the laminar convective heat transfer with vapor condensation [27], the total viscous dissipation after optimization only increased by $3.35 \%$, while the total heat transfer rate increased by $31.67 \%$ and the condensing heat transfer rate increased by $29.26 \%$. For the same heat transfer increment, the viscous dissipation increment for turbulent heat transfer with condensation was much larger than that for laminar convective heat transfer with condensation. The reason for this is that turbulent heat transfer is much stronger than laminar convection. Therefore, more pumping power should be put into further enhancement of turbulent heat transfer with condensation. For the optimized case, any variation in the optimal flow field will lead to a reduction of heat transfer rate for a given pumping power.

The velocity vectors at the cross section $z=30 \mathrm{~mm}$ in the tube are shown in Figure 3(a). Local distributions of the temperature and mass fraction of the vapor near the tube surface are shown in Figure 3(b) and (c). It can be seen that the optimal flow pattern has multiple longitudinal vortices near the tube surface, which significantly affect the distributions of temperature and vapor concentration.

Compared with the case before optimization, the temperature and vapor concentration gradients increased in the region where the flow contacted the tube surface. The synergy angles between the velocity vector and the temperature gradient decreased, as did the synergy angles between the velocity vector and the vapor concentration gradient. The synergies not only between the velocity and temperature fields but also between the velocity and vapor concentration fields were improved. Therefore, the turbulent heat and mass transfer were enhanced. However, in the region where the flow was away from the tube surface, the temperature and vapor concentration gradients decreased. The synergy angles between the velocity vector and temperature gradient increased, as did the synergy angles between the velocity vector and the vapor concentration gradient. The synergies not only between the velocity and temperature fields, but also between the velocity and vapor concentration fields were reduced. For the whole tube, the flow with multiple longitudinal vortices near the tube surface significantly improved the synergies not only between the velocity and temperature fields, but also between the velocity and vapor concentration fields, leading to enhanced turbulent heat and mass transfer.

For turbulent heat transfer with condensation, the temperature and vapor concentration gradients near the tube surface were much larger than those in the core region. Heat and mass transfer resistances mainly exist in the viscous sublayer. Therefore, flow with multiple longitudinal vortices near the tube surface can reduce the thickness of the viscous sublayer, resulting in reduced heat and mass transfer resistances and enhanced turbulent heat and mass transfer. The thickness of the viscous sublayer for fully developed turbulent flow with $R e=10000$ is $0.32 \mathrm{~mm}$. Figure 3 shows that the distance between the vortex center and the tube surface was about $0.9 \mathrm{~mm}$, which is about 3 times as

Table 1 Optimization results for flue gas turbulent heat transfer with condensation

\begin{tabular}{ccc}
\hline & $\begin{array}{c}\text { Before } \\
\text { optimization }\end{array}$ & $\begin{array}{c}\text { After } \\
\text { optimization }\end{array}$ \\
\hline Time-averaged viscous dissipation (W) & $4.73 \times 10^{-3}$ & $1.02 \times 10^{-2}$ \\
Total heat transfer rate (W) & 39.65 & 51.78 \\
Condensing heat transfer rate (W) & 28.32 & 40.20 \\
\hline
\end{tabular}



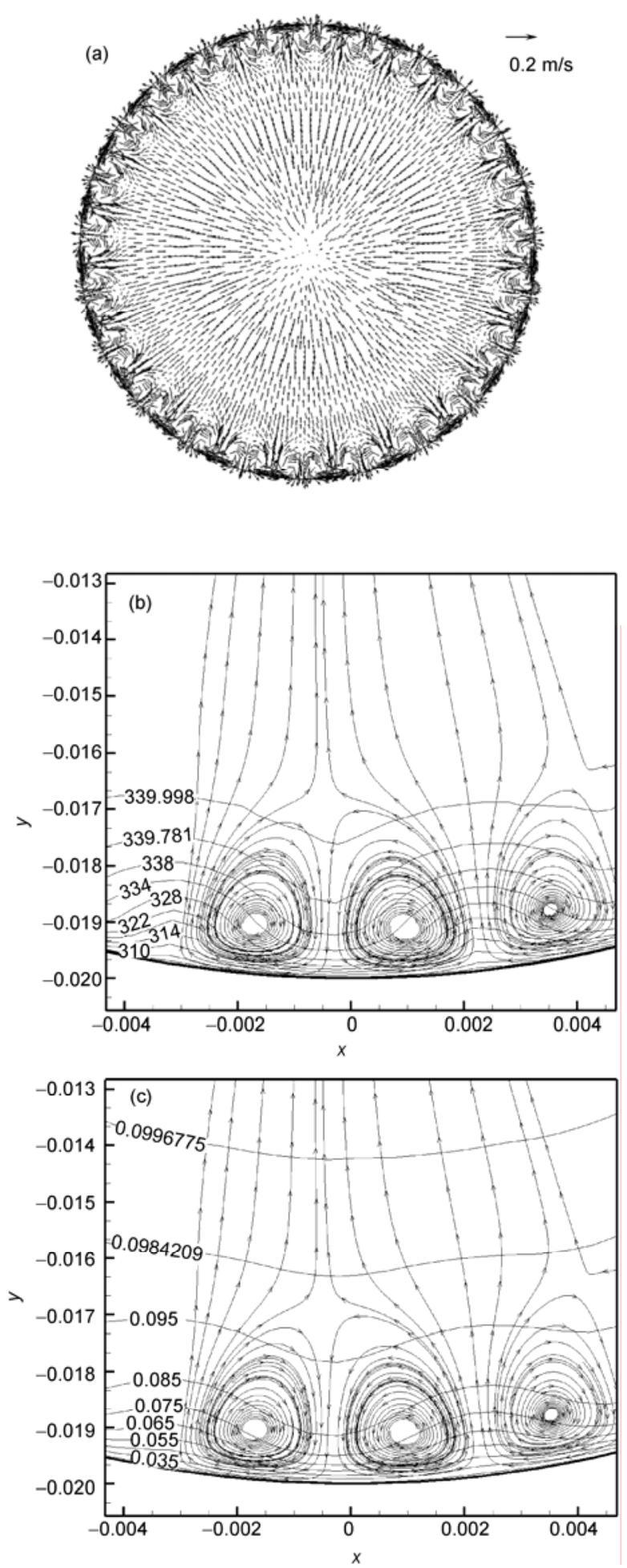

Figure 3 Optimization results for the flue gas turbulent heat transfer with condensation. (a) Velocity vectors; (b) temperature distribution (unit: K); (c) mass fraction of the vapor.

the thickness of the viscous sublayer, while during optimization of the laminar convective heat transfer with vapor condensation [27], the vortices took up almost the entire flow channel, and the distance between the vortex center and the channel wall was half the height of the channel.

For practical application, a series of small longitudinal vortex generators on the tube surface can be used to generate multiple longitudinal vortices, similar to the optimal flow, thereby enhancing the turbulent heat transfer with condensation.

\section{Conclusions}

Entransy and entransy dissipation were defined for turbulent heat transfer with vapor condensation. The field synergy equation for the turbulent heat transfer with condensation was deduced using the EED principle.

Numerical results show that the optimal flow pattern has multiple longitudinal vortices, which improve the synergies not only between the velocity and temperature fields, but also between the velocity and vapor concentration fields. Therefore, flue gas turbulent heat transfer with condensation can be enhanced by longitudinal vortices with given timeaveraged viscous dissipation. For practical application, flow with multiple longitudinal vortices, similar to the optimal velocity field, can be generated to enhance the turbulent heat transfer with condensation.

This work was supported by the National Basic Research Program of China (G2007CB206901).

1 Zhang X H, Liu D W. Evaluation of reclaiming technology for gas-fired boiler flue gas residual heat (in Chinese). Industrial Boiler, 2008, 4: 4-8

2 Jia L, Peng X F, Sun J D, et al. Theoretical analysis on heat transfer in flue gas with vapor condensation (in Chinese). J Therm Sci Tech, 2002, 1: 15-19

3 Jia L, Peng X F. Research on convective condensation heat transfer for mixture gas in a vertical tube (in Chinese). Industrial Heating, 2002, 5: 28-30

4 Li X P, Jia L, Sun J D. An experimental study on convection condensing heat transfer of wet flue gas in a vertical tube (in Chinese). J Beijing Institute Eng Arch, 2002, 18: 19-23

5 Jia L, Peng X F, Yan Y, et al. Effects of water vapor condensation on the convection heat transfer of wet flue gas in a vertical tube. Int $\mathbf{J}$ Heat Mass Transfer, 2001, 44: 4257-4265

6 Jia L, Peng X F. Heat transfer in flue gas with vapor condensation. Tsinghua Sci Tech, 2002, 7: 177-181

7 Jia L, Peng X F. Research on convective condensation heat transfer for a gas mixture in a vertical tube. Heat Transfer-Asian Res, 2004, 33: 219-228

8 Bejan A. Advanced Engineering Thermodynamics. New York: Wiley, 1997

9 Guo Z Y, Zhu H Y, Liang X G. Entransy-A physical quantity describing heat transfer ability. Int J Heat Mass Transfer, 2007, 50: 2545-2556

10 Guo Z Y, Liang X G, Zhu H Y. Entransy-A physical quantity describing heat transfer ability (in Chinese). Pro Natural Sci, 2006, 16: 1288-1296

11 Xia S J, Chen L G, Sun F R. Entransy dissipation minimization for liquid-solid phase processes. Sci China: Tech Sci, 2010, 53: 960-968

12 Xiao Q H, Chen L G, Sun F R. Constructal entransy dissipation rate and flow-resistance minimizations for cooling channels. Sci China: 
Tech Sci, 2010, 53: 2458-2468

13 Xie Z H, Chen L G, Sun F R. Constructal optimization on T-shaped cavity based on entransy dissipation minimization. Chinese Sci Bull, 2009, 54: 4418-4427

14 Wei S H, Chen L G, Sun F R. Constructal optimization of discrete and continuous variable cross-section conducting path based on entransy dissipation rate minimization. Sci China: Tech Sci, 2010, 53: 1666-1677

15 Xie Z H, Chen L G, Sun F R. Constructal optimization of twice level Y-shaped assemblies of fins by taking maximum thermal resistance minimization as objective. Sci China: Tech Sci, 2010, 53: 2756-2764

16 Xia S J, Chen L G, Sun F R. Optimization for entransy dissipation minimization in heat exchanger. Chinese Sci Bull, 2009, 54: 3587-3595

17 Wu J, Cheng X G, Meng J A, et al. Potential capacity dissipation extremum and entropy generation minimazation in laminar convective heat transfer (in Chinese). J Eng Thermophys Chin, 2006, 27: 100-102

18 Chen Q, Wu J, Ren J X. Thermodanamic optimization and heat transfer optimization for convective heat transfer (in Chinese). J Eng Thermophys Chin, 2008, 29: 271-274

19 Liu X B, Meng J A, Guo Z Y. Entropy generation extremum and entransy dissipation extremum for heat exchanger optimization. Chinese Sci Bull, 2009, 54: 943-947

20 Chen Q, Wang M R, Pan N, et al. Optimization principles for con- vective heat transfer. Energy, 2009, 34: 1199-1206

21 Liu X B, Guo Z Y. A novel method for heat exchanger analysis (in Chinese). Acta Phys Sin, 2009, 58: 4766-4771

22 Chen L, Chen Q, Li Z, et al. Optimization for a heat exchanger couple based on the minimum thermal resistance principle. Int $\mathbf{J}$ Heat Mass Transfer, 2009, 52: 4778-4784

23 Meng J A, Liang X G, Li Z X. Field synergy optimization and enhanced heat transfer by multi-longitudinal vortices flow in tube. Int $\mathrm{J}$ Heat Mass Transfer, 2005, 48: 3331-3337

24 Song W M, Meng J A, Li Z X. Optimal velocity field for laminar convective heat transfer in rectangular channel (in Chinese). J Eng Thermophys Chin, 2011, 32: 133-136

25 Chen Q, Ren J X, Meng J A. Field synergy equation for turbulent heat transfer and its application. Int J Heat Mass Transfer, 2007, 50: 5334-5339

26 Chen Q, Yang K D, Wang M R, et al. A new approach to analysis and optimization of evaporative cooling system I: Theory. Energy, 2010, 35: 2448-2454

27 Song W M, Meng J A, Li Z X. Optimization of flue gas convective heat transfer with condensation in a rectangular channel. Chinese Sci Bull, 2011, 56: 263-268

28 Chen $\mathrm{Q}, \mathrm{Xu} \mathrm{W}$. A zero-equation turbulence model for indoor air flow simulation. Energ Buildings, 1998, 28: 137-144

29 Zhao B, Li X, Yan Q. A simplified system for indoor airflow simulation. Build Environ, 2003, $38: 543-552$

Open Access This article is distributed under the terms of the Creative Commons Attribution License which permits any use, distribution, and reproduction in any medium, provided the original author(s) and source are credited. 